\title{
The effectiveness and safety of moxibustion for treating cancer-related fatigue: a systematic review and meta-analyses
}

\author{
Seunghoon Lee • Ui Min Jerng • Yan Liu • \\ Jung Won Kang • Dongwoo Nam • Jae-dong Lee
}

Received: 24 July 2013 / Accepted: 3 February 2014 / Published online: 8 March 2014

(C) Springer-Verlag Berlin Heidelberg 2014

\begin{abstract}
Purpose Among cancer patients, cancer-related fatigue (CRF) is one of the most common symptoms and adversely affects physical ability and quality of life even several years after treatment. This study aims to evaluate the current evidence for moxibustion in patients with CRF.

Methods Eighteen databases were searched from their inception to April 2013. All randomized controlled trials (RCTs) of moxibustion for treating CRF without language restriction were considered for inclusion. The risk of bias and reporting quality of each study were assessed using the Cochrane risk of bias tool, Consolidated Standards of Reporting Trials (CONSORT), and Revised Standards for Reporting Interventions in Clinical Trials of Acupuncture (STRICTA). Risk ratio (RR) or mean difference (MD) was used to measure the treatment effect with $95 \%$ confidence intervals (CIs) in a random effects model.

Results Four RCTs with a total of 374 subjects were included for the review. These four studies compared moxibustion plus routine care with routine care alone. Most studies were determined to have a moderate to high risk of bias with low reporting quality. An indirect moxa stick was used in two studies, an
\end{abstract}

Electronic supplementary material The online version of this article (doi:10.1007/s00520-014-2161-z) contains supplementary material, which is available to authorized users.

S. Lee $\cdot$ J. W. Kang $\cdot$ D. Nam $\cdot$ J.-d. Lee $(\bowtie)$

Department of Acupuncture \& Moxibustion Medicine, College of

Korean Medicine, Kyung Hee University, Seoul, South Korea

e-mail: 1jdacu@gmail.com

\section{U. M. Jerng}

Department of Oncology, College of Korean Medicine, Kyung Hee University, Seoul, South Korea

S. Lee $\cdot$ Y. Liu

Acupuncture, Moxibustion \& Meridian Research Group, Korea Institute of Oriental Medicine, Daejeon, South Korea indirect ginger cake-separated moxa was used in one study, and in one remaining study, both moxibustion methods were used. Meta-analysis showed the favorable effects of moxibustion on the response rate (RR, $1.73 ; 95 \% \mathrm{CI}, 1.29$ to $2.32 ; p=.0003$; heterogeneity, $I^{2}=15 \%, p=.32$ ). Burning with a mild blister after moxibustion was reported in one study.

Conclusions Because of a high risk of bias and low reporting quality of the studies included in this review, it is difficult to draw the conclusion that moxibustion is an effective and safe treatment for patients with $\mathrm{CRF}$. Further rigorous research will be necessary to evaluate whether moxibustion has beneficial effects on CRF.

Trial registration PROSPERO. Unique identifier: CRD42013004501.

Keywords Cancer-related fatigue $\cdot$ Moxibustion $\cdot$ Systematic review $\cdot$ Meta-analysis

\section{Introduction}

Fatigue is the most commonly reported symptom in patients with cancer, distinct from the type of tiredness felt by healthy individuals after daily life activities [1,2]. Cancer-related fatigue (CRF) is defined as a "persistent, subjective sense of tiredness related to cancer and cancer treatment that interferes with usual functioning" by the National Comprehensive Cancer Network (NCCN) [3]. Among cancer patients, CRF considerably affects the functional status and health-related quality of life (QoL) with reduction of ability to perform physical, mental, emotional, or social functions [1, 4].

Although the prevalence of CRF varies according to the definition or clinical status of cancer, most studies have suggested that the proportion of patients with cancer who complain of generalized fatigue is in excess of $60 \%[5,6]$. In one study with 379 cancer patients having undergone chemotherapy, 
$91 \%$ of the subjects complained that fatigue negatively affects their daily lives [7]. It is also reported that this fatigue persists for years in approximately $30 \%$ of breast cancer survivors [8].

The etiology of CRF is poorly understood, but is presumed to be a combination of both physiological factors (e.g., anemia, cancer treatment, tumor burden, and cachexia) and psychosocial factors (e.g., anxiety, depression, and sleep disturbance) [1]. Some immunogenomic markers such as TNF- $\alpha$, IL-1b, IL-2, and IL-6 are known to play a major role as physiological initiators of CRF $[2,4]$.

$\mathrm{CRF}$ is observed in cancer patients with various clinical statuses. Fatigue occurs mainly during active cancer treatments, such as cytotoxic chemotherapy, radiation therapy, or immunotherapy, but it can persist for several months or years in posttreatment patients even after the malignancy is cured because of persistent activation or late effects of cancer treatments $[1,7,9]$. Advanced cancer patients in terminal stage can become more easily fatigued because disease progression increases fatigue [10]. These different clinical statuses may influence symptom severity and management strategies [10].

Current management of CRF includes nonpharmacologic treatments (e.g., psychosocial interventions, exercise, sleep therapy, or acupuncture) and pharmacologic treatments (e.g., stimulants, antidepressants, or steroids) [11]. One clinical guideline [10] recommends selecting these interventions according to the patient's clinical status. In recent Cochrane systematic reviews, some of the pharmacologic treatments were regarded as a beneficial option for CRF [12]. However, and because these medications have unsatisfactory effects with potential adverse outcomes, an increasing number of patients experiencing CRF have been seeking complementary and integrative medicine.

Moxibustion is a traditional therapy from East Asian countries which applies the heat of burning herbs, primarily Artemisia vulgaris, to stimulate specific spots on the skin [13]. In addition to acupuncture, moxibustion has been used for palliative cancer care, as well as CRF [14]. Although moxibustion and acupuncture have similarities and are sometimes used in combination, there are differences in treatment methods and mechanisms between these two modalities. Acupuncture is performed by insertion of needles with specific manipulation, such as twisting or thrusting the needle inducing a de qi sensation, to induce biochemical response in the soft tissue. On the other hand, moxibustion treatment works through direct or indirect thermal stimulation at various temperature levels $[13,15]$. It has been reported that this thermal stimulation on specific acupuncture points reduces oxidative stress [16] and improves the immunosuppressive state $[17,18]$ in animal models. According to one randomized controlled trial (RCT), indirect moxibustion at CV4 and CV8 improved chronic fatigue with modification of the antioxidant activity when compared to the placebo control [19]. These effects have been proposed as potential mechanisms by which moxibustion appears to improve chronic fatigue, including CRF $[2,20,21]$.

In one prospective study with 523 cancer patients in Korea [22], $55 \%$ of patients who were diagnosed with cancer began complementary therapy within 3 years of cancer diagnosis. Another cross-sectional study with 1,150 cancer patients [23] reported that $75.0 \%$ of the participants used complementary and alternative medicine (CAM), and $21.9 \%$ of these patients sought CAM for the treatment of CRF. Moreover, $13.9 \%$ of cancer patients who presented to one Korean medical clinic were treated with moxibustion for cancer-related symptoms, including CRF [23]. Recently, three systematic reviews have been published on effectiveness and safety of acupuncture $[24,25]$ and CAM [26] for CRF. However, there is no systematic review focusing on moxibustion currently. Therefore, this study aims to review the evidence for effectiveness and safety of moxibustion for treating CRF.

The objective of the present review is to assess whether moxibustion (a) is more effective and safer compared to waiting list/no treatment, (b) has a greater efficacy than a placebo, (c) is more effective and safer compared to routine care, and (d) is more effective and safer when it is used as an adjuvant therapy to routine care than routine care alone for reducing CRF in cancer patients.

\section{Methods}

Protocol and registration

The protocol of the review methods was registered prospectively (CRD42013004501; http://www.crd.york.ac.uk/ PROSPERO).

\section{Data sources}

\section{Electronic searches}

The following 18 databases were searched from inception through to April 2013: MEDLINE (1946 to April Week 2 2013), EMBASE (1980 to April 2, 2013), the Cochrane Central Register of Controlled Trials (The Cochrane Library, 2013 issue 3), the Cumulative Index to Nursing and Allied Health Literature (CINAHL, 1982 to April 2013), Allied and Complementary Medicine Database (AMED, 1985 to April 2013), PsycINFO (1806 to April Week 2 2013), three Chinese databases (China National Knowledge Infrastructure (CNKI), Wanfang, VIP), one Japanese database (Japan Science and Technology Information Aggregator, Electronic (J-STAGE)), and eight Korean databases (Korean Association of Medical Journal Edition (KAMJE), Korean Medical Database (KMBASE), Korean Studies Information Service System (KISS), National Discovery for Science Leaders (NDSL), 
Database Periodical Information Academic (DBpia), Korean National Assembly Digital Library (KNADL), Oriental Medicine Advanced Searching Integrated System (OASIS), and Korean Traditional Knowledge Portal (KTKP)). The WHO International Clinical Trials Registry Platform (ICTRP) was also searched for ongoing and recently completed studies. The search terms consisted of three parts: Cancer (e.g., cancer, neoplasm, or tumor), Fatigue (e.g., fatigue, tiredness, or weary), and Moxibustion (e.g., moxibustion, moxa, mugwort, or wormwood). The detailed search strategies for MEDLINE, Chinese databases, and Korean databases are shown in Online Resource 1.

\section{Searching other resources}

Bibliographic references in relevant publications (e.g., oncology textbooks on integrative medicine and CAM, clinical guidelines of CRF, other review articles, and included clinical trials) were manually searched to avoid missing eligible trials otherwise not referenced in databases.

Study selection

\section{Types of studies}

RCTs of moxibustion treatment for treating CRF were included for this review, as either full articles or abstracts. Nonrandomized controlled trials, uncontrolled clinical trials (e.g., case studies), and qualitative studies were excluded. No language or publication status restrictions were applied. In randomized cross-over trials, only data from the first period would be included to avoid the carry-out effect.

\section{Types of participants}

All patients experiencing CRF were included, without restrictions on cancer type, status of severity, and duration of cancer and fatigue. Additionally, the patient's clinical status (e.g., during active treatment, posttreatment, or at terminal stage) was not restricted, but the results according to the different statuses were described separately.

\section{Types of interventions}

Moxibustion treatment using any types of stimulations (direct, indirect, heat-sensitive, moxa burner, or natural moxibustion) [27] was included. Trials using acupuncture or herbal medicine as a co-intervention were also included because moxibustion treatment is often accompanied by these interventions in practice. Additionally, trials using moxibustion as an adjuvant therapy to other active treatment were included. However, trials were excluded if moxibustion was a minor component of treatment option in the trial. We included trials comparing moxibustion to waiting list/no treatment, sham (placebo) moxibustion, and routine care (e.g., education, physical therapies, psychosocial interventions, or conventional medications). Trials in which one form of moxibustion was compared with another were excluded.

\section{Types of outcome measures}

We included only those studies that assessed CRF using validated scales as a main outcome. Performance status, QoL, effects on immunity, and adverse events were assessed as secondary outcomes. Studies which evaluated only these secondary outcomes were excluded.

Data collection and analysis

\section{Selection of studies}

Each of the three review authors (SL, UMJ, and YL) independently screened the titles and abstracts of studies identified by the search. Each article was reviewed for study selection by every member, and the decision to include or exclude a given study was recorded on a standard eligibility form. If disagreements regarding a selection of a study could not be resolved through discussion, the final decision was left for the arbiter (KHK) who is expert on this field.

\section{Data extraction and management}

Three review authors (SL, UMJ, and YL) read the full text of articles selected for review and independently extracted the data using a standard data extraction form. Any disagreement among the authors was resolved by discussion. If reported data were insufficient or ambiguous, the author (YL) who is fluent in the Chinese and English languages contacted the corresponding authors through e-mail or by telephone to request additional information or clarification.

\section{Assessment of risk of bias and reporting quality in included studies}

Three review authors (SL, UMJ, and YL) independently evaluated the risk of bias based on the "Risk of bias" tool developed by the Cochrane Collaboration. Quality of reporting was assessed using the Consolidated Standards of Reporting Trials (CONSORT) and the Standards for Reporting Interventions in Clinical Trials of Acupuncture (STRICTA).

\section{Quantitative data synthesis}

For dichotomous outcomes, the risk ratio (RR) was used to measure the treatment effect with $95 \%$ confidence intervals 
(CIs). For continuous data, either the mean difference (MD) or, if different methods or scales are used for calculating the same outcome variables, the standardized mean difference (SMD) was used to measure the treatment effect with $95 \%$ CIs. Random-effects model with $95 \%$ CIs was preferred for quantitative synthesis of clinical outcomes due to the expected clinical heterogeneity of included trials. In cases of considerable heterogeneity $\left(I^{2}>75 \%\right)$ that could not be explained by the diversity in clinical or methodological features among the included trials, we did not pool the data [28], and the possible reasons for the inconsistency are described in the "Discussion" section. Studies included for meta-analysis were pooled as RRs. For pooled data, the Review Manager (RevMan) software (version 5.2 for Windows; the Nordic Cochrane Centre, Copenhagen, Denmark) was used for calculating summary statistics. When there was an adequate number of studies, we conducted subgroup analysis to interpret the heterogeneity between studies according to type of moxibustion (e.g., direct, indirect, heat-sensitive, moxa burner, or natural moxibustion), type of control (e.g., waiting list/no treatment, sham/placebo moxibustion, or routine care), and whether the patient did or did not receive chemotherapy or radiation therapy. When possible, sensitivity analyses were performed to determine whether the results were robust according to sample size (e.g., more or less than 30 participants in each group), methodological qualities (e.g., allocation concealment or participant/assessor blinding), and analysisrelated issues (e.g., processes to handle missing data).

\section{Results}

Of the 1,022 articles identified, 1,000 were excluded upon identifying exclusion criteria in the title or abstract. Of the remaining 22, 18 additional studies were excluded upon having reviewed the main body of the text, leaving four eligible RCTs [29-32] with a total of 374 patients (see Fig. 1). Data regarding trial characteristics and moxibustion and control interventions are described in Table 1. All four RCTs had been performed in China.

\section{Study description}

\section{Participants}

Two RCTs [29, 30] included patients with stage III and IV of various cancers, and one RCT [32] did not restrict cancer stage or type in patient selection. The remaining study [31] included patients with stage III and IV diseases, but did not report cancer type. Three trials [29-31] included patients who had completed cancer treatment. Two of these trials [29,31] excluded patients who had received radiotherapy and/or chemotherapy within 4 weeks before participating in the trials, and one trial [30] excluded patients who had received radiotherapy and/or chemotherapy during treatment. All RCTs included only inpatients admitted to a cancer specialty hospital.

\section{Moxibustion interventions}

All of the RCTs had used a combination of indirect moxibustion and routine care. Two RCTs [29, 30] used moxa sticks, one [31] applied ginger cake-separated moxas, and the remaining study [32] used both moxibustion methods. In all of the RCTs, the selection of the acupuncture point for moxibustion treatment had been based on the traditional Chinese medicine (TCM) theory, and the rationale behind the selection was described in each article. The time of each moxibustion treatment ranged from 5 to $30 \mathrm{~min}$, and the total number of treatment sessions varied from 14 to 40 . Two RCTs [29, 31] used fixed acupuncture points, and the others [30, 32] used both fixed and individual points determined by the practitioner. The details of moxibustion regimens are summarized in Table 2.

\section{Controls}

All of the RCTs had used routine care as a control. A control in one RCT [30] included nutritional counseling, regular exercises, and Chinese herb decoctions. In one RCT [32], the control treatment included education, psychological supportive care, appropriate activity, exercise, diet, and adequate sleep. There was no detailed description of control treatment in the remaining RCTs $[29,31]$.

\section{Risk of bias assessment}

Two RCTs [29, 30] employed appropriated sequence generation methods for randomization using a random number table, but none of the studies had reported details regarding allocation concealment. Blinding of participants was impossible because there was no trial comparing with sham moxibustion. One RCT [29] reported their study design to be singleblinded, but there was no detailed description. The risk of bias for reporting dropout and withdrawal was high in one RCT [32], and there was a considerable difference in dropout rates between groups (moxibustion group, 27 (37\%); control, 7 $(10 \%))$. Three RCTs [29-31] did not report dropout or withdrawal participants. None of the RCTs mentioned if the data were analyzed by intention-to-treat (ITT) analysis. The risk of bias for selective outcome reporting and other sources of bias were unclear for all four RCTs. We judged that most trials had a moderate to high risk of bias (see Table 3).

\section{Reporting quality}

Because all of the trials included in this review had been conducted after 2010 and published in 2012, the revised 
Fig. 1 Flow chart of report selections. $C C T$ controlled clinical trial, $C R F$ cancer-related fatigue, $R C T$ randomized clinical trial, UCT uncontrolled clinical trial

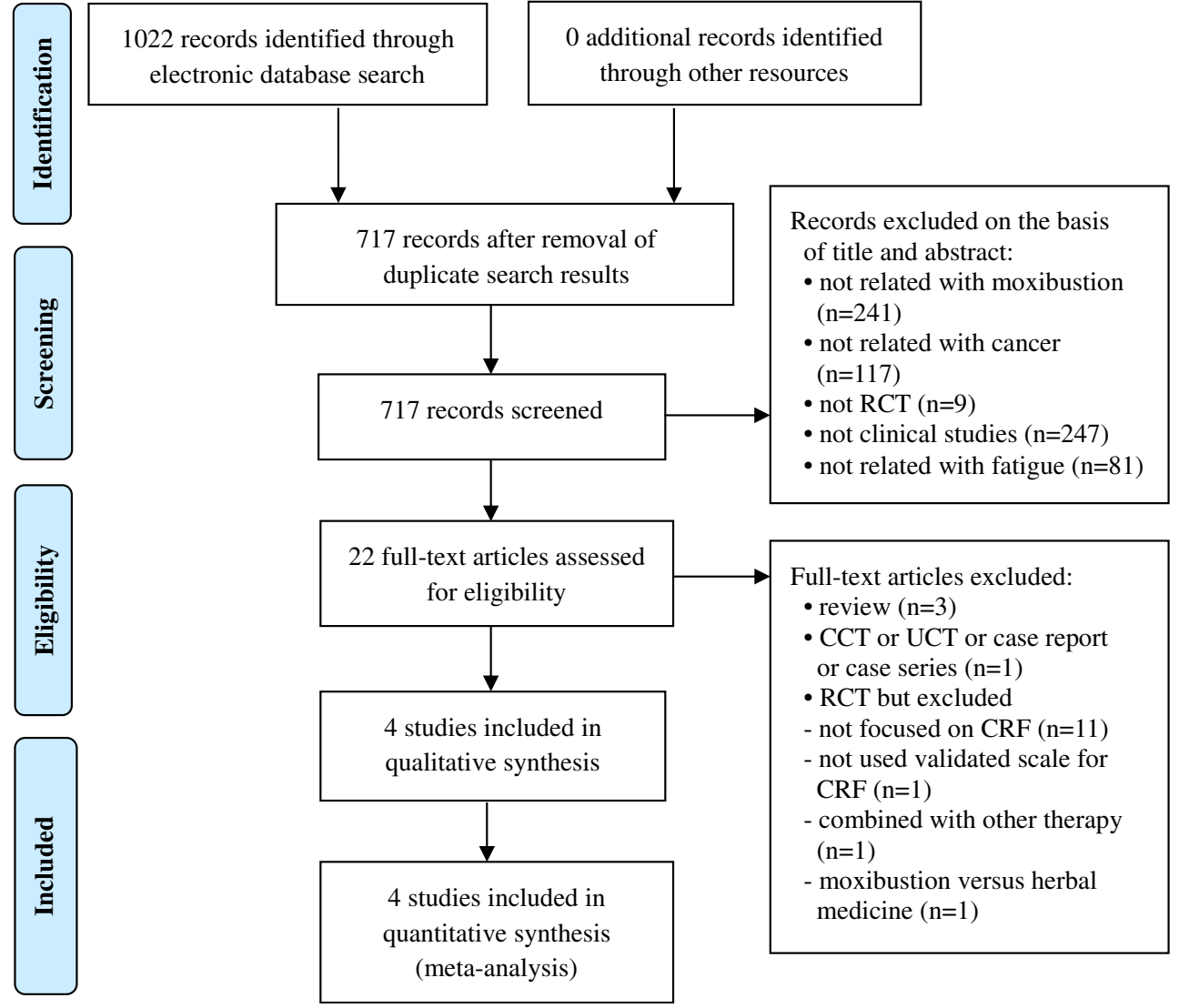

version of the CONSORT 2010 statement and the new STRICTA recommendation were used to assess the quality of the included RCTs. Based on the 25 standards of the CONSORT statement, all the RCTs described eligibility criteria for participants and trial setting through which each study was carried out. Basic statistical methods to compare groups were documented in all articles. Two RCTs [30, 32] indicated baseline demographic and clinical characteristics in a table. Only one RCT [30] explained the scientific background and rationale and reported adverse events. None of the articles adequately reported the following: methods of random allocation concealment, sample size estimate, definition of the prespecified primary outcome, addressing the potential limitation of the study design, and preregistration in a clinical trial registry. The detailed results are presented in Online Resource 2.

All RCTs were also evaluated based on the 6 items and 17 sub-items of STRICTA. Three items (style of moxibustion, reasoning for choosing treatment details, and treatment regimen respectively corresponding with STRICTA $1 \mathrm{a}, 1 \mathrm{~b}$, and 3) were well-stated in all articles. Only two RCTs [30, 32] described the feature of moxibustion, procedure and technique of moxibustion treatment, and control treatment in detail. The background of the practitioner and information to patients were not described in any of the articles. These findings are presented in Online Resource 3.

\section{Details of included trials}

Qin et al. [29] evaluated the clinical curative effect of warm moxibustion for fatigue in patients with advanced stages of cancer. Seventy-eight patients were divided randomly into two parallel groups: the treatment group received moxibustion treatment (5-10 min once daily for 20 days, a total two sessions, $n=36$ ) plus routine care, and the control group received only routine care $(n=42)$. The outcome measurement of CRF was the Piper Fatigue Scale (PFS), which was converted to three categorical data (no/mild, moderate, or severe). At the conclusion of this trial, $75 \%$ (27 out of 36 ) of the patients experienced no/mild fatigue in the treatment group, compared to $36 \%$ (15 out of 42 ) of the patients in the control group $(p<.05)$.

Another trial conducted by Qin et al. [30] aimed to assess the effect of moxibustion at the abdomen and lower limbs on CRF with immunologic assessment. Thirty inpatients were randomized into treatment group (moxibustion with symptomatic treatment, $n=15$ ) and control group (only symptomatic treatment, $n=15$ ). Moxibustion treatment was conducted for $30 \mathrm{~min}$ once daily for 14 days, and symptomatic treatment included nutritional counseling, regular exercise, and Chinese herb decoctions. In the treatment group, $40 \%$ (6 out of 15) of the patients experienced no/mild fatigue at the primary endpoint, and none 


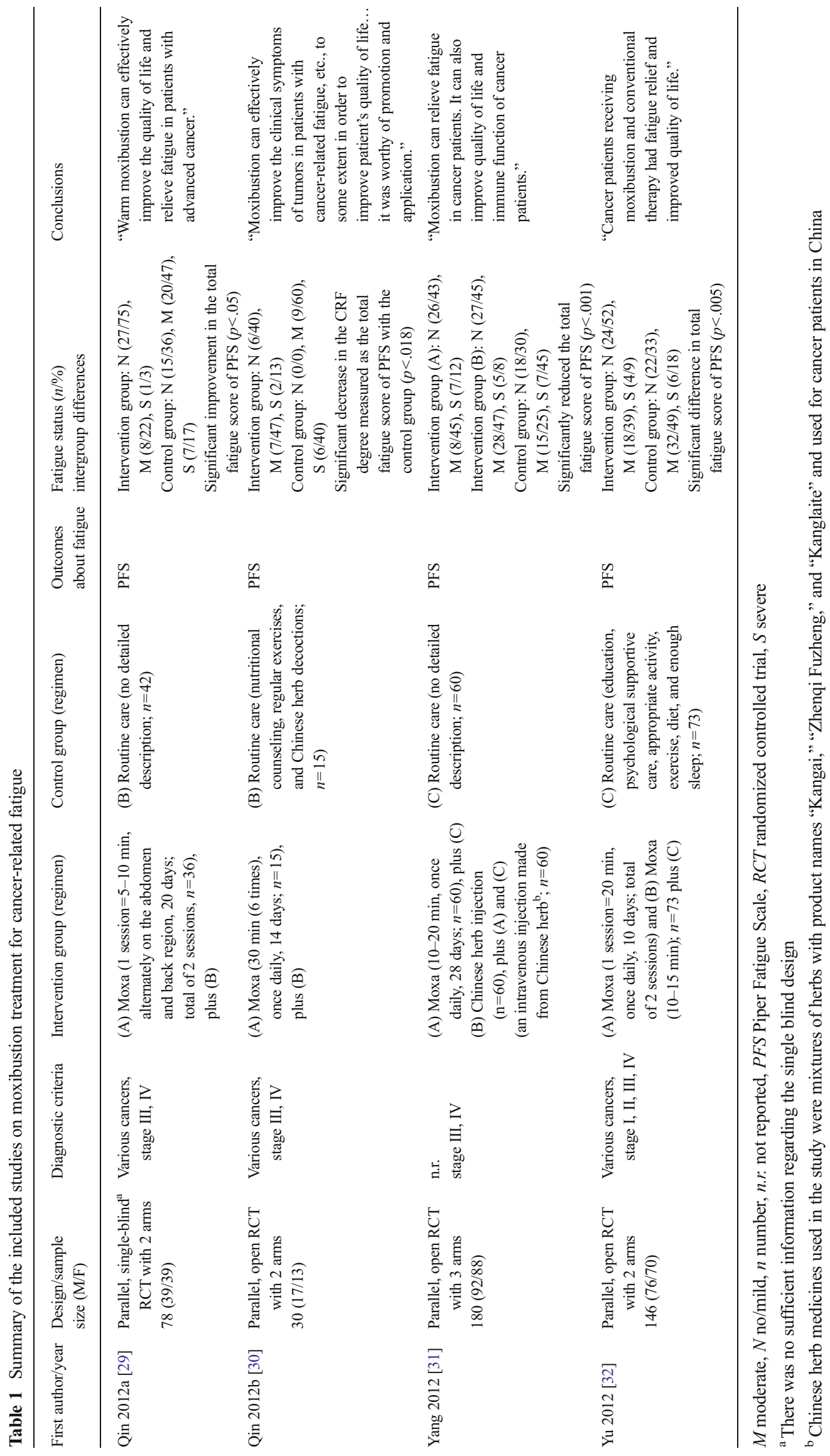




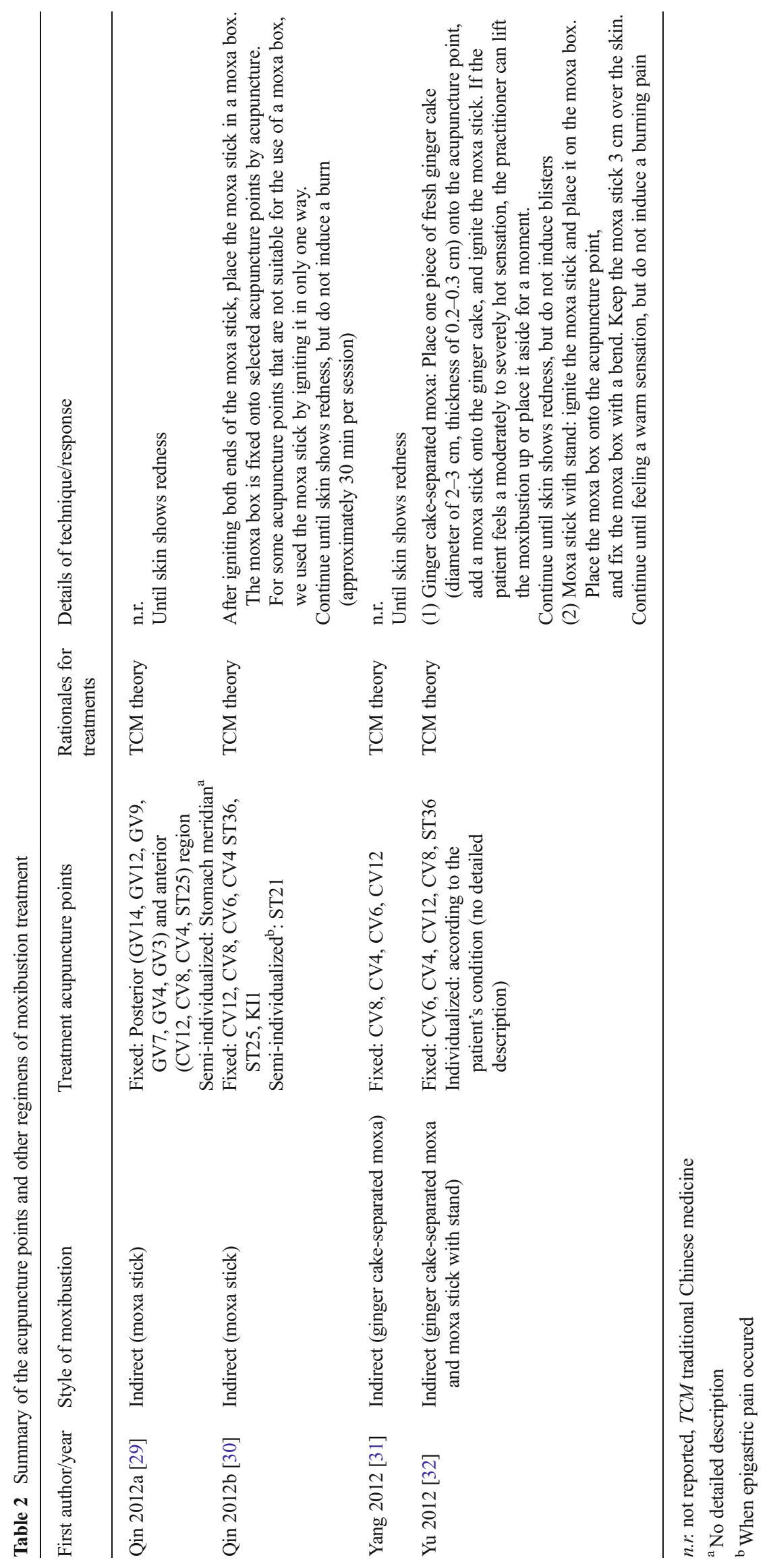


Table 3 Risk of bias in the randomized clinical trials

\begin{tabular}{llllllll}
\hline First author/year & $\begin{array}{l}\text { Sequence } \\
\text { generation }\end{array}$ & $\begin{array}{l}\text { Allocation } \\
\text { concealment }\end{array}$ & $\begin{array}{l}\text { Patient } \\
\text { blinding }\end{array}$ & $\begin{array}{l}\text { Assessor } \\
\text { blinding }\end{array}$ & $\begin{array}{l}\text { Incomplete data } \\
\text { addressed }\end{array}$ & $\begin{array}{l}\text { Selective outcome } \\
\text { reporting }\end{array}$ & $\begin{array}{l}\text { Other source } \\
\text { of bias }\end{array}$ \\
\hline Qin 2012a [29] & $\mathrm{L}$ & $\mathrm{U}$ & $\mathrm{H}$ & {$[\mathrm{U}]$} & {$[\mathrm{U}]$} & $\mathrm{U}$ & $\mathrm{U}$ \\
Qin 2012b [30] & $\mathrm{L}$ & $\mathrm{U}$ & $\mathrm{H}$ & $\mathrm{U}$ & {$[\mathrm{U}]$} & $\mathrm{U}$ & $\mathrm{U}$ \\
Yang 2012 [31] & $\mathrm{U}$ & $\mathrm{U}$ & $\mathrm{H}$ & $\mathrm{U}$ & {$[\mathrm{U}]$} & $\mathrm{U}$ & $\mathrm{U}$ \\
Yu 2012 [32] & $\mathrm{U}$ & $\mathrm{U}$ & $\mathrm{H}$ & $\mathrm{U}$ & $\mathrm{H}$ & $\mathrm{U}$ \\
\hline
\end{tabular}

We judged the quality assessment of each domain based on the Cochrane risk of bias tool. For domains enclosed in brackets, we contacted the respective authors because of an ambiguous address to ask for additional data but received no response

$H$ high risk of bias, $L$ low risk of bias, $U$ unclear risk of bias

of the patients in the control group had no/mild fatigue $(p=.018)$.

Yang et al. [31] conducted a three-armed open trial to evaluate moxibustion and Chinese herb. The control group $(n=60)$ received routine care, and the two treatment groups were as follows: the moxibustion group $(n=60)$ which received 10-20 min of ginger cake-separated moxa treatment once daily for 28 days plus routine care, and the moxibustioncombined Chinese herb medicine group $(n=60)$ which received a Chinese herb injection for 28 days in addition to the treatments that the moxibustion group received. The number of patients with severe CRF, as measured by PFS, was significantly lower in both moxibustion group (26 out of 60 , $p<.001)$ and moxibustion-combined Chinese herb medicine group (27 out of $60, p<.001$ ), when compared to the control group (18 out of $60, p>.05$ ). The authors stated that the moxibustion-combined Chinese herb injection was more effective for CRF than the moxibustion-only treatment.

Yu et al. [32] aimed to determine whether moxibustion treatment reduces CRF versus routine care. One hundred forty-six patients were randomly divided into two groups: the moxibustion group $(n=73)$ which received both indirect moxa sticks (20 min, once daily for 20 days) and ginger cakeseparated moxas (10-15 min) plus routine care and a control group $(n=73)$ which received only routine care (education, psychological supportive care, appropriate activities, exercise, diet, and adequate sleep). The authors reported that the proportions of patients who experienced no/mild fatigue were
$52 \%$ (24 out of 46$)$ in the treatment group and $33 \%(22$ out of 66) in the control group $(p<.05)$.

\section{Outcomes}

\section{Quantitative synthesis of the main outcome}

In all of the RCTs, fatigue was measured by PFS, which is a multidimensional instrument developed to evaluate fatigue in cancer patients and consists of 22 items and 4 subscales [33]. Because the total fatigue score of PFS is presented as ordinal variables converted to severity codes $(0=$ none, $1-3=$ mild, 4 $6=$ moderate, and $7-10=$ severe), we converted these categories to dichotomous outcomes such as responder (none and mild) or nonresponder (moderate and severe) in the data pool.

The meta-analysis of included RCTs showed favorable effects of moxibustion on the response rate $(n=340$; RR, 1.73; $95 \%$ CI, 1.29 to $2.32 ; p=.0003$; heterogeneity, $I^{2}=$ $15 \%, p=.32, X^{2}=3.53$; Fig. 2). None of the RCTs had followed up the patients after the moxibustion treatment period. Subgroup and sensitivity analyses were not performed owing to a lack of studies included in the review.

\section{Other outcomes}

Two RCTs [30, 31] compared moxibustion plus routine care with routine care alone by assessing performance status with the Karnofsky Performance Status (KPS) scale. In one

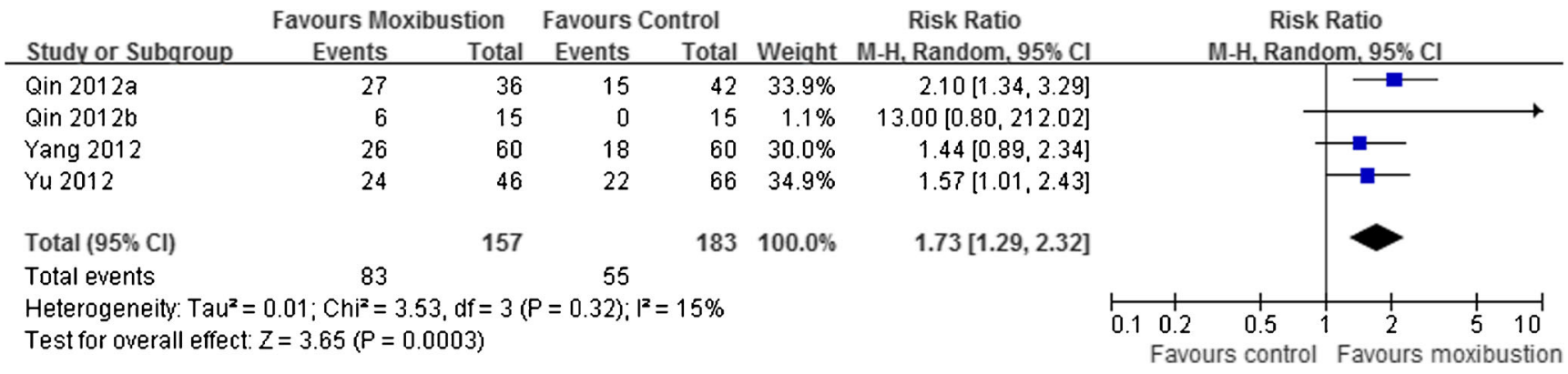

Fig. 2 A forest plot of the effectiveness of moxibustion for treating cancer-related fatigue on response rate 
study [30], a significant improvement in KPS score was observed in the moxibustion plus routine care group compared to the routine care alone group (MD, 13.23; $95 \% \mathrm{CI}, 6.91$ to 19.55). The other study [31] did not provide detailed data (e.g., mean and SD) but reported significant improvements in physical performance in the moxibustion plus routine care group compared to the routine care alone group $(p<.05)$.

One RCT [29] evaluated global health status with EORTCQLQ-C30 which is a questionnaire developed to assess the QoL of cancer patients. This study compared moxibustion treatment plus routine care to routine care alone and showed a significant improvement in global health status after moxibustion treatment (MD, 5.57; $95 \%$ CI, 3.41 to 7.73).

Two RCTs [30, 31] used biomarkers to assess immunological responses after moxibustion treatment. One RCT [30] measured total T cell, T helper cell (Th cell), suppressor T cell (Ts cell), and natural killer (NK) cell counts and determined the $\mathrm{CD} 4+/ \mathrm{CD} 8+$ ratio. After 14 days of moxibustion treatment, the Ts cell count was slightly reduced (MD, $-0.09 ; 95 \%$ $\mathrm{CI},-3.30$ to 3.12 ), and the T cell (MD, 3.04; $95 \% \mathrm{CI},-8.36$ to 14.44), Th cell (MD, 1.10; $95 \% \mathrm{CI},-1.58$ to 3.78 ), and NK cell (MD, 0.90; $95 \% \mathrm{CI},-3.41$ to 5.21 ) counts and the CD4+/ $\mathrm{CD} 8+$ ratio (MD, $0.09 ; 95 \% \mathrm{CI},-0.23$ to 0.41 ) had increased; however, these changes were not statistically significant. The other RCT [31] measured total T cell, Th cell, Ts cell, and NK cell counts. After 28 days of moxibustion treatment, total $\mathrm{T}$ cell (MD, 9.95; $95 \%$ CI, 8.62 to 11.28), Th cell (MD, 6.90; $95 \%$ CI, 5.73 to 8.07), and NK cell (MD, 7.59; $95 \%$ CI, 6.23 to 8.28 ) counts were significantly increased.

\section{Adverse events}

One RCT [30] reported a single case of burning, with a mild blister, after moxibustion treatment, which resolved without a scar after 2 days. None of the trials reported any serious adverse reaction from moxibustion treatment.

\section{Discussion}

The objective of this systematic review was to evaluate the effectiveness and safety of moxibustion in the treatment of CRF. All four trials reported a noticeable improvement in CRF in the groups receiving moxibustion treatment plus routine care compared to the control groups receiving routine care alone. This finding was confirmed by the current metaanalysis. Despite the overall positive results across all included trials, the high risk of bias and the low number of eligible RCTs made it difficult to find conclusive evidence of the effectiveness of moxibustion as a treatment for CRF. Additionally, only one trial mentioned adverse events associated with moxibustion, which was the single case of burning, with a mild blister, which lasted for 2 days. For this reason, a conclusion regarding the safety of moxibustion treatment could also not be reached because of insufficient evidence.

While acupuncture [24, 25] and other CAM [26] trials for CRF have reported contradictory results, all trials in this review reported a noticeable improvement in CRF with moxibustion therapy. However, it is necessary to interpret this result cautiously because of the limitations in each of the included studies. Some factors may have contributed to the overestimation of the actual therapeutic effects. Firstly, the frequency of treatment sessions may have influenced the results. All four trials used moxibustion treatment daily. This frequency of treatment was more than that of acupuncture trials on CRF [24] or that of other moxibustion trials on chronic fatigue [19]. It is supposed that the increased frequency of treatments was possible because most trials [30, 31, 32] recruited hospital inpatients only. A systematic review of irritable bowel syndrome found that trials with an increased frequency of treatments were more likely to report positive outcomes in Chinese-language literature [34]. As all the studies in our meta-analysis were on Chinese populations with increased frequency of treatments, this tendency might explain the overly positive outcomes in this review. Secondly, subjective and patient-centered outcomes are easily affected by patient expectations and preferences. Fatigue is usually assessed by patient-centered questionnaires because fatigue itself is a subjective symptom that is difficult to measure with an objective assessment tool. Patients who favored moxibustion treatment were more likely to be recruited in these trials, but patients in the respective control groups received only routine care, which was also offered to the moxibustion group. These might have led participants in the moxibustion group to reply more positively compared to the control group. Other trials in which other subjective outcomes (e.g., symptom severity or QoL) were measured also tended to have the same problem [34]. Thirdly, none of the trials included in this study used sham-controlled placebo devices to assess the specific effect of moxibustion. Some placebo moxibustions [35-37] have been developed to produce the heat sensation of moxibustion, and some clinical studies [19, 38] have used these sham devices as placebo. However, it is unlikely that a proper placebo moxibustion will be developed because the heating effect from a sham device may not be inert, which is a similar problem encountered with sham acupuncture. For this reason, most moxibustion trials have used routine care as a control [39-41]. Performance bias is inevitable in moxibustion trials as long as these active controls are used without an inert placebo. Because none of the trials included in this review were designed to distinguish the nonspecific effect of moxibustion, the effect size of the moxibustion group can easily be shown to be higher than that of the control group. Finally, most trials did not present baseline data appropriately. Baseline imbalance is known to be a potential important threat to internal validity and can be sufficient to cause an 
exaggeration of effect estimates [28]. In particular, the effect of CRF treatments is more likely to be affected by baseline data such as patient factors (e.g., performance status or fatigue severity) and disease factors (e.g., cancer stage) [42, 43]. However, half of the included studies in this review $[31,32]$ did not present detailed baseline data. The study by $\mathrm{Yu}$ et al. [32] did not report the fatigue severity and cancer stage of each group, although they reported that the severity of fatigue was highly related to the cancer stage at baseline. Moreover, it is known that an improper randomization process can easily result in baseline imbalance [28], but none of the included studies in this review were free from the bias of random sequence generation and allocation concealment.

It was also difficult to determine the safety of moxibustion because of a lack of evidence in the trials. Moxibustion treatment requires more caution of the operator than acupuncture treatment because it can easily induce a dermal burn. A cross-sectional study reported that the incidence of adverse events by moxibustion treatment in clinical trials was higher than that by acupuncture treatment (16.8\% vs $5.1 \%$ ) [44]. The most common adverse event in moxibustion trials was reported to blistering caused by burning. Among all trials included in this review, only one trial [30] mentioned one case of burning with a blister as an adverse event. Although indirect moxibustion induces less adverse events than direct moxibustion, the total incidence of adverse events in all included trials is too low. Moreover, the smoke from moxibustion may be harmful if inhaled by cancer patients. Since patients with primary or metastatic tumors in the lung often complain of dyspnea [45, 46], moxibustion treatment could potentially have a negative impact on these cancer patients. Because all RCTs in this review recruited stage IV patients, and three trials $[29,30,32]$ included primary lung cancer patients, it is possible that the patients from these trials had pulmonary symptoms including dyspnea. However, none of the patients mentioned respiratory complications. Considering poor reporting quality in most trials, it is difficult to suggest that each investigator examined adverse events closely. Before moxibustion can be considered safe for CRF, a strict study measuring expectable adverse events is required.

This review has several limitations. Firstly, all included studies were Chinese trials published in acupuncture journals. A recent methodology study found that most RCTs published in certain Chinese journals did not adequately address randomization and the allocation concealment procedure, and many non-RCTs were published as RCTs with a high risk of bias [47]. None of the trials included in our review described the method of concealment in detail, and only two trials mentioned generation of a random sequence [29, 30]. Moreover, because all trials were conducted in China, the results are difficult to generalize to other populations. Secondly, the specific effect of moxibustion in each trial was difficult to determine because no trial compared moxibustion to a placebo control. In addition to the difficulty of using a sham control in moxibustion trials, the high prevalence and cultural preference for moxibustion in China make detection of a real benefit difficult. Thirdly, most trials included in this review were regarded to have a high risk of bias and methodological flaws; no trials had a low risk of bias in all domains. Furthermore, reporting quality assessed by CONSORT and STRICTA was too low to draw a meaningful conclusion.

With strict regard to the limitations of individual trials, we recommend certain methodological improvements for future studies evaluating the use of moxibustion for patients with CRF. Firstly, it is necessary to consider including adequate blinding methods. Because the use of placebo controls in oncology trials is controversial in view of the ethical ramifications, many oncology trials have forgone the use of a placebo. In order to adapt placebo controls to oncology trials, some methodological and ethical criteria need to be fulfilled [48]. Fatigue is a symptom with a high placebo response rate, so placebo controls in clinical trials for CRF may be justified methodologically. Additionally, if the placebo group receives enhanced routine care or conventional medications offered to all groups and includes a smaller proportion of patients than the treatment groups, the ethical issues will be diminished somewhat. Therefore, if an appropriate model of sham moxibustion is developed, it is recommended to use a placebo control with blinding of study assessors to identify the true efficacy of moxibustion for treating CRF. Secondly, it is necessary to conduct an objective assessment of fatigue in addition to using the subjective outcomes. As fatigue is easily affected by patient preference and expectations, it is recommended to use semi-subjective outcomes, such as loss of working days due to fatigue, change in the amount of other conventional treatment, or economic evaluation, as well as subjective outcomes $[49,50]$. Because recent trials reported that moxibustion has a therapeutic effect for some diseases with improvement of the immune response, biomarkers for immunity should be evaluated. Thirdly, methods that are more proper are needed to evaluate the symptoms associated with CRF. A number of unidimensional (e.g., EORTC-QLQ C30 fatigue subscale and the Functional Assessment of Cancer Therapy Fatigue (FACT-F)) and multidimensional scales (e.g., PFS and the Multidimensional Fatigue Inventory (MFI)) have been developed to measure the severity of CRF $[51,52]$. One systematic review for the measurement of CRF [51] demonstrated that multidimensional scales are limited in their usage for clinical trials because most multidimensional scales require complex administration and an extensive amount of time and still have insufficient data (less than 1,000 patients). Moreover, the advantages of additional dimensional measuring of CRF (e.g., "cognitive," "physical," or "emotional" fatigue) are not clear [53]. All included studies in this review used PFS, which is a multidimensional scale that has only little or no data in most types of cancer patients, 
except for breast cancer patients. Also, PFS was validated in only active treatment patients and disease-free survivors and was not validated in posttreatment patients [33]. For these reasons, further studies need to measure cancer-related fatigue with well-validated unidimensional scales for all types of cancer and clinical status, such as EORTC-QLQ C30 or FACT-F [51]. Finally, further trials should have a low risk of bias and adequately report methods and results according to CONSORT and STRICTA guidelines. It has been demonstrated that adequate allocation is the most critical source of bias in RCTs [54]. Recent well-designed systematic review of acupuncture for chronic pain only included trials which used unambiguous methods of allocation concealment [55]. But because adequacy of allocation concealment has not been clear in moxibustion trials to date, allocation concealment should be performed adequately and reported precisely. Further trials designed with statistical accuracy are also needed because none of the RCTs in this review was conducted according to appropriate statistical methods, such as sample size calculation and ITT analysis. Moreover, proper evaluation and description of adverse events are necessary to recommend clinical guidelines with a comparison of the benefits and harms of moxibustion treatment for CRF.

In conclusion, there is limited evidence indicating that moxibustion may be effective for improving fatigue in cancer patients. However, the studies meeting our inclusion criteria had small sample sizes, low methodological quality, and a high risk of bias, therefore no concrete recommendation can be made for the generalized use of moxibustion in patients with CRF. A large-scale RCT with high-quality methodology is warranted for further evaluation of this treatment modality.

Acknowledgments SL was supported by the Korea Institute of Oriental Medicine (K13011). We acknowledge Professor Kun Hung Kim for providing helpful suggestions.

Conflict of interest The authors have no conflicts of interest to disclose.

\section{References}

1. Ahlberg K, Ekman T, Gaston-Johansson F, Mock V (2003) Assessment and management of cancer-related fatigue in adults. Lancet 362(9384):640-650. doi:10.1016/s0140-6736(03)14186-4

2. Ryan JL, Carroll JK, Ryan EP, Mustian KM, Fiscella K, Morrow GR (2007) Mechanisms of cancer-related fatigue. Oncologist 12(Suppl 1):22-34. doi:10.1634/theoncologist.12-S1-22

3. Mock V, Atkinson A, Barsevick A, Cella D, Cimprich B, Cleeland C, Donnelly J, Eisenberger MA, Escalante C, Hinds P, Jacobsen PB, Kaldor P, Knight SJ, Peterman A, Piper BF, Rugo H, Sabbatini P, Stahl C, National Comprehensive Cancer N (2000) NCCN practice guidelines for cancer-related fatigue. Oncology 14(11A):151-161
4. Saligan LN, Kim HS (2012) A systematic review of the association between immunogenomic markers and cancer-related fatigue. Brain Behav Immun 26(6):830-848. doi:10.1016/j.bbi.2012.05.004

5. Cella D, Davis K, Breitbart W, Curt G, Fatigue C (2001) Cancerrelated fatigue: prevalence of proposed diagnostic criteria in a United States sample of cancer survivors. J Clin Oncol 19(14): 3385-3391

6. Stone P (2002) The measurement, causes and effective management of cancer-related fatigue. Int J Palliat Nurs 8(3):120-128

7. Curt GA, Breitbart W, Cella D, Groopman JE, Horning SJ, Itri LM, Johnson DH, Miaskowski C, Scherr SL, Portenoy RK, Vogelzang NJ (2000) Impact of cancer-related fatigue on the lives of patients: new findings from the Fatigue Coalition. Oncologist 5(5):353-360

8. Lindley C, Vasa S, Sawyer WT, Winer EP (1998) Quality of life and preferences for treatment following systemic adjuvant therapy for early-stage breast cancer. J Clin Oncol 16(4):1380-1387

9. Bower JE, Ganz PA, Aziz N, Fahey JL, Cole SW (2003) T-cell homeostasis in breast cancer survivors with persistent fatigue. $\mathrm{J}$ Natl Cancer Inst 95(15):1165-1168

10. Berger AM, Abernethy AP, Atkinson AA, Breitbart WS, Braothers B, Cella D, Cleeland C, Eisenberger MA, Escalante CP, Jacobsen PB, Kaldor P, Ligibel JA, Loggers ET, Mandrell B, Mooney K, Murphy BA, O'Connor T, Pirl WF, Rugo HS, Wagner LI, Wagner-Johnston ND (2014) NCCN clinical practice guidelines in oncology (NCCN Guidelines ${ }^{\circledR}$ ): cancer-related fatigue. Version 1. http://www.nccn.org/ professionals/physician_gls/PDF/fatigue.pdf. Accessed 19 Jan 2014

11. Escalante CP, Manzullo EF (2009) Cancer-related fatigue: the approach and treatment. J Gen Intern Med 24(Suppl 2):S412-416. doi: 10.1007/s11606-009-1056-Z

12. Minton O, Richardson A, Sharpe M, Hotopf M, Stone P (2010) Drug therapy for the management of cancer-related fatigue. Cochrane Database Syst Rev 7, CD006704. doi:10.1002/14651858. CD006704.pub3

13. World Health Organization (2007) WHO international standard terminologies on traditional medicine in the Western Pacific Region. World Health Organization, Western Pacific Region

14. Kuai L, Chen H, Yang HY (2008) Current status and prospect of acupuncture-moxibustion in treatment of cancer pain: a review. Zhong Xi Yi Jie He Xue Bao 6(2):197-202

15. Kim JI, Choi JY, Lee H, Lee MS, Ernst E (2010) Moxibustion for hypertension: a systematic review. BMC Cardiovasc Disord 10:33. doi:10.1186/1471-2261-10-33

16. Hua JS, Li LP, Zhu XM (2008) Effects of moxibustion pretreating on SOD and MDA in the rat of global brain ischemia. J Tradit Chin Med 28(4):289-292

17. Pei J, Wei H, Liu ZD, Yu YM, Ni CR, Wu HG (2010) Effects of moxibustion on the expression of IL-1beta, IL-2, IL-6 mRNA and protein in the cerebral cortex in tumor-bearing mice. Zhen Ci Yan Jiu 35(4):243-249

18. Tang Z, Song X, Li J, Hou Z, Xu S (1996) Studies on antiinflammatory and immune effects of moxibustion. Zhen Ci Yan Jiu 21(2):67-70

19. Kim HG, Yoo SR, Park HJ, Son CG (2013) Indirect moxibustion (CV4 and CV8) ameliorates chronic fatigue: a randomized, doubleblind, controlled study. J Altern Complement Med 19(2):134-140. doi:10.1089/acm.2011.0503

20. Bounous G, Molson J (1999) Competition for glutathione precursors between the immune system and the skeletal muscle: pathogenesis of chronic fatigue syndrome. Med Hypotheses 53(4):347-349. doi:10. 1054/mehy.1998.0780

21. Maes M, Mihaylova I, De Ruyter M (2006) Lower serum zinc in chronic fatigue syndrome (CFS): relationships to immune dysfunctions and relevance for the oxidative stress status in CFS. J Affect Disord 90(2-3):141-147. doi:10.1016/j.jad.2005.11.002

22. Kim SG, Park EC, Park JH, Hahm MI, Lim JH, Choi KS (2007) Initiation and discontinuation of complementary therapy among 
cancer patients. J Clin Oncol 25(33):5267-5274. doi:10.1200/ jco. 2007.11.9651

23. Chang S-B, Lee T-W, Kim S, Yoo I-Y, Kim I-S, Kang K-H, Lee M-K, Jang Y-H (2006) A study of complementary and alternative medicine used by cancer patients in Korea. Korean J Adult Nurs 18(1):92-101

24. Posadzki P, Moon TW, Choi TY, Park TY, Lee MS, Ernst E (2013) Acupuncture for cancer-related fatigue: a systematic review of randomized clinical trials. Support Care Cancer 21(7):2067-2073. doi: 10.1007/s00520-013-1765-Z

25. Garcia MK, McQuade J, Haddad R, Patel S, Lee R, Yang P, Palmer JL, Cohen L (2013) Systematic review of acupuncture in cancer care: a synthesis of the evidence. J Clin Oncol 31(7):952-960. doi:10. $1200 /$ jco. 2012.43 .5818

26. Finnegan-John J, Molassiotis A, Richardson A, Ream E (2013) A systematic review of complementary and alternative medicine interventions for the management of cancer-related fatigue. Integr Cancer Ther. doi:10.1177/1534735413485816

27. Cheng CW, Fu SF, Zhou QH, Wu TX, Shang HC, Tang XD, Liu ZS, Liu J, Lin ZX, Lao L, Lu AP, Zhang BL, Liu BY, Bian ZX (2013) Extending the CONSORT statement to moxibustion. J Integr Med 11(1):54-63. doi:10.3736/jintegrmed2013009

28. Higgins JPT, Green S, Cochrane C (2008) Cochrane handbook for systematic reviews of interventions. Wiley-Blackwell, Chichester

29. Qin X, Liu Z (2012) Clinical observation of wen moxibustion therapy cancer-related fatigue of advanced cancer. China J Chin Med 27(166):273-274

30. Qin X (2012) Clinical research on the effect of moxibustion on cancer-related fatigue. Guanzhou University of Chinese Medicine

31. Yang JL, Yu L, Xu L, Wang J, Jing NC (2012) The effectiveness of moxibustion treatment to treat cancer-related fatigue for elderly patients with end stage cancer. Chin J Gerontol 32:4408-4410, In Chinese

32. Yu L, Jing NC, Yang JL, Hou YY, Xu L (2012) Clinical feature and observation of moxibustion treatment for cancer-related fatigue. Guide China Med 31:591-593, In Chinese]

33. Piper BF, Dibble SL, Dodd MJ, Weiss MC, Slaughter RE, Paul SM (1998) The revised Piper Fatigue Scale: psychometric evaluation in women with breast cancer. Oncol Nurs Forum 25(4):677684

34. Manheimer E, Wieland LS, Cheng K, Li SM, Shen X, Berman BM, Lao L (2012) Acupuncture for irritable bowel syndrome: systematic review and meta-analysis. Am J Gastroenterol 107(6):835-847. doi: 10.1038/ajg.2012.66, quiz 848

35. Zhao B, Wang X, Lin Z, Liu R, Lao L (2006) A novel sham moxibustion device: a randomized, placebo-controlled trial. Complement Ther Med 14(1):53-60. doi:10.1016/j.ctim.2005.06. 003, discussion 61

36. Jang MK, Yoon EH, Jung CY, Byun H, Kim EJ, Kim KH, Kim KS, Lee SD (2010) Credibility of a newly developed sham moxibustion. J Korean Acupunct Moxibustion Soc 27(1):117-127

37. Kim SY, Yi SH, Cho JH, Yin CS, Lee H, Park HJ (2011) Heat stimulation on the skin for medical treatment: can it be controlled? J Altern Complement Med 17(6):497-504. doi:10.1089/acm.2010. 0072

38. Park JE, Sul JU, Kang K, Shin BC, Hong KE, Choi SM (2011) The effectiveness of moxibustion for the treatment of functional constipation: a randomized, sham-controlled, patient blinded, pilot clinical trial. BMC Complement Altern Med 11:124. doi:10.1186/14726882-11-124
39. Choi TY, Choi J, Kim KH, Lee MS (2012) Moxibustion for the treatment of osteoarthritis: a systematic review and meta-analysis. Rheumatol Int 32(10):2969-2978. doi:10.1007/s00296-012-2367-7

40. Lee MS, Choi TY, Park JE, Ernst E (2010) Effects of moxibustion for constipation treatment: a systematic review of randomized controlled trials. Chin Med 5:28. doi:10.1186/1749-8546-5-28

41. Lee MS, Shin BC, Kim JI, Han CH, Ernst E (2010) Moxibustion for stroke rehabilitation: systematic review. Stroke 41(4):817-820. doi: 10.1161/strokeaha.109.566851

42. Lawrence DP, Kupelnick B, Miller K, Devine D, Lau J (2004) Evidence report on the occurrence, assessment, and treatment of fatigue in cancer patients. JNCI Monogr 32:40-50. doi:10.1093/ jncimonographs/lgh027

43. Kim D-I, Choi M-S (2013) Guidance for prospective acupuncture treatment on cancer-related fatigue (CRF). Ann Palliat Med 2(1):710

44. Jung HJ, Park JE, Liu Y, Kim AR, Choi SM (2012) The analysis of incidence and type of adverse events in acupuncture \& moxibustion clinical trials. Korean J Acupunct 29(3):421-430

45. Bruera E, Schmitz B, Pither J, Neumann CM, Hanson J (2000) The frequency and correlates of dyspnea in patients with advanced cancer. J Pain Symptom Manag 19(5):357-362

46. Temel JS, Pirl WF, Lynch TJ (2006) Comprehensive symptom management in patients with advanced-stage non-small-cell lung cancer. Clin Lung Cancer 7(4):241-249. doi:10.3816/CLC.2006.n.001

47. Wu T, Li Y, Bian Z, Liu G, Moher D (2009) Randomized trials published in some Chinese journals: how many are randomized? Trials 10:46. doi:10.1186/1745-6215-10-46

48. Daugherty CK, Ratain MJ, Emanuel EJ, Farrell AT, Schilsky RL (2008) Ethical, scientific, and regulatory perspectives regarding the use of placebos in cancer clinical trials. J Clin Oncol 26(8):13711378. doi:10.1200/jco.2007.13.5335

49. MacPherson H, Bland M, Bloor K, Cox H, Geddes D, Kang'ombe A, Reynolds J, Stamuli E, Stuardi T, Tilbrook H, Torgerson D, Whorwell P (2010) Acupuncture for irritable bowel syndrome: a protocol for a pragmatic randomised controlled trial. BMC Gastroenterol 10:63. doi:10.1186/1471-230x-10-63

50. Xue CC, Helme RD, Gibson S, Hogg M, Arnold C, Somogyi AA, Da Costa C, Wang Y, Lu SC, Zheng Z (2012) Effect of electroacupuncture on opioid consumption in patients with chronic musculoskeletal pain: protocol of a randomised controlled trial. Trials 13:169. doi:10.1186/1745-6215-13-169

51. Minton O, Stone P (2009) A systematic review of the scales used for the measurement of cancer-related fatigue (CRF). Ann Oncol 20(1): 17-25. doi:10.1093/annonc/mdn537

52. Jean-Pierre P, Figueroa-Moseley CD, Kohli S, Fiscella K, Palesh OG, Morrow GR (2007) Assessment of cancer-related fatigue: implications for clinical diagnosis and treatment. Oncologist 12(Suppl 1):1121. doi:10.1634/theoncologist.12-S1-11

53. Lai JS, Crane PK, Cella D (2006) Factor analysis techniques for assessing sufficient unidimensionality of cancer related fatigue. Qual Life Res 15(7):1179-1190. doi:10.1007/s11136-006-0060-6

54. Schulz KF, Chalmers I, Hayes RJ, Altman DG (1995) Empirical evidence of bias. Dimensions of methodological quality associated with estimates of treatment effects in controlled trials. JAMA 273(5): 408-412

55. Vickers AJ, Cronin AM, Maschino AC, Lewith G, MacPherson H, Foster NE, Sherman KJ, Witt CM, Linde K, Acupuncture Trialists C (2012) Acupuncture for chronic pain: individual patient data metaanalysis. Arch Intern Med 172(19):1444-1453. doi:10.1001/ archinternmed.2012.3654 